\title{
Unexpected diversity of CRISPR unveils some evolutionary patterns of repeated sequences in Mycobacterium tuberculosis
}

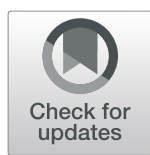

\author{
Guislaine Refrégier $^{1 *}$, Christophe Sola ${ }^{1 *}$ (D) and Christophe Guyeux ${ }^{2}$
}

\begin{abstract}
Background: Diversity of the CRISPR locus of Mycobacterium tuberculosis complex has been studied since 1997 for molecular epidemiology purposes. By targeting solely the 43 spacers present in the two first sequenced genomes ( $H 37 R v$ and BCG), it gave a biased idea of CRISPR diversity and ignored diversity in the neighbouring cas-genes.

Results: We set up tailored pipelines to explore the diversity of CRISPR-cas locus in Short Reads. We analyzed data from a representative set of 198 clinical isolates as evidenced by well-characterized SNPS.

We found a relatively low diversity in terms of spacers: we recovered only the 68 spacers that had been described in 2000. We found no partial or global inversions in the sequences, letting always the Direct Variant Repeats (DVR) in the same order. In contrast, we found an unexpected diversity in the form of: SNPs in spacers and in Direct Repeats, duplications of various length, and insertions at various locations of the IS6110 insertion sequence, as well as blocks of DVR deletions. The diversity was in part specific to lineages. When reconstructing evolutionary steps of the locus, we found no evidence for SNP reversal. DVR deletions were linked to recombination between IS6110 insertions or between Direct Repeats.

Conclusion: This work definitively shows that CRISPR locus of M. tuberculosis did not evolve by classical CRISPR adaptation (incorporation of new spacers) since the last most recent common ancestor of virulent lineages. The evolutionary mechanisms that we discovered could be involved in bacterial adaptation but in a way that remains to be identified.
\end{abstract}

\section{Background}

Since the rise of molecular biology, repeated sequences (CRISPR, IS, VNTRs) have been used to track relatedness between individuals [1]. Indeed, they share two major features essential for diversity studies: ease of study, and rapid mutation rate [2]. In pathogens like Mycobacterium tuberculosis complex (MTC) they have been used for molecular epidemiology, complementing contact tracing, and/or identifying unsuspected links [1]. In the last 5 years

\footnotetext{
* Correspondence: guislaine.refregier@u-psud.fr; christophe.sola@universiteparis-saclay.fr

${ }^{1}$ Institute for Integrative Biology of the Cell (I2BC), CEA, CNRS, Univ. Paris-Sud, Université Paris-Saclay, cedex, 91198 Gif-sur-Yvette, France

Full list of author information is available at the end of the article
}

however, popularity of most repeated sequences has decreased first because they are larger than reads provided by Short Reads Sequencing, and second because of the generalization of Whole-Genome-Sequence availability and use of softwares analyzing Single Nucleotide Polymorphisms (SNPs) [3-5]. In fact, some of these repeated sequences have sufficient variation to characterize them based on reads. The boom of Whole Genome Sequencing provides plenty of data to dig into for evolutionary studies and changes the way drug-susceptibility testing will be done in the future $[6,7]$. We will show in the case of CRIS PR sequences how this diversity can reveal unexpected evolutionary patterns. We will show in addition that in the species of focus, namely MTC, there has been no new

C C The Author(s). 2020 Open Access This article is licensed under a Creative Commons Attribution 4.0 International License, which permits use, sharing, adaptation, distribution and reproduction in any medium or format, as long as you give appropriate credit to the original author(s) and the source, provide a link to the Creative Commons licence, and indicate if changes were made. The images or other third party material in this article are included in the article's Creative Commons licence, unless indicated otherwise in a credit line to the material. If material is not included in the article's Creative Commons licence and your intended use is not permitted by statutory regulation or exceeds the permitted use, you will need to obtain permission directly from the copyright holder. To view a copy of this licence, visit http://creativecommons.org/licenses/by/4.0/ The Creative Commons Public Domain Dedication waiver (http://creativecommons.org/publicdomain/zero/1.0/) applies to the data made available in this article, unless otherwise stated in a credit line to the data. 
spacers acquisition for at least 5000 years, i.e. no adaptative evolution in the common CRISPR terminology despite the presence of Cas genes.

CRISPR acronym stands for Clustered Regularly Interspaced Short Palindromic Repeats [8]. They are characterized by repeats of 21 to $37 \mathrm{bp}$ called Direct Repeats (DR) and the presence of unique sequences, called spacers, between each DR copy. Blocks of one DR and the following spacer has been termed Direct Variable Repeat (DVR) [9]. CRISPR loci were first identified in Escherichia coli [10], their role in bacterial immunity was suspected in Yersinia pestis [11], and later demonstrated in Streptococcus thermophilus [12]. Their presence has been detected in around $50 \%$ percent of eubacteria and $90 \%$ of archaebacteria [13-17]. Various classes of CRISPR systems have been described [18]. They all share the same mechanism of spacer acquisition, inserting part of a foreign sequence designated as protospacer, with a length similar to that of the repeats, next to the $5^{\prime}$ end of the locus. In Salmonella enterica for instance, the exploration of CRISPR diversity has shown that sequences including several DVR could be deleted, and that mutations could occur in spacers [19], however, the increased CRISPR dictionary as well as the restricted number of genomes sequenced reduced the possibility to have an extensive understanding of their evolutionary mechanisms.

Mycobacterium tuberculosis complex (MTC) is the agent of mammal tuberculosis, with human-adapted lineages being the most diverse and well spread. Its emergence and diversification dates back to at least 5000 years old. There are six main and widely spread humanadapted sublineages referred to as L1 to L6 and an animal-adapted lineage [20-23], as well as a few rare and endemic human lineages (L7, L8, L0) [24, 25]. Their diversity is being progressively unveiled through extensive WGS [20, 21, 26, 27].

M. tuberculosis reference clinical isolate $\mathrm{H} 37 \mathrm{Rv}$ as well as most $M$. tuberculosis isolates carry a CRISPR locus together with a complete cas genes set of type III-A [18]. Rare isolates lack part of CRISPR and or cas genes [28]. Partial analysis of the CRISPR diversity has been used since 1997 to explore the clinical isolates relatedness through a technique coined as « spoligotyping » [29]. In this technique, the presence of 43 spacers identified in H37Rv $(n=35)$ or in M. bovis BCG $(n=8)$ are looked for. This results in a barcode that can be easily shared and stored. Spoligotyping has led to the set-up of the first worldwide database for this pathogen counting today more than 111,000 patterns originating from 169 countries [13, 14]. The absence in some isolates of individual or consecutive spacers has revealed the possibility for small and large deletions of adjacent DVR [30, 31]. Large deletions proved good markers of tuberculosis diversification [32,33].
Extensive MTC CRISPR structure has been previously explored in $19 \mathrm{M}$. tuberculosis clinical isolates belonging to EAI (L1), Beijing (L2), Euro-American (L4) lineages, five from animal species $M$. bovis and M. microti, and one $M$. canettii [34]. This work showed that additional diversity exists in the form of DR variants, and duplication of DVR. It also documented the presence of insertion sequence IS6110 in two different positions and orientations in L2 and L4 lineages. CRISPR diversity however remains unexplored in many sublineages as well as in major lineages such as L3, L5 and L6.

We recently set up a pipeline to reconstruct reliably CRISPR locus of M. tuberculosis [35]. We selected Short Reads Archives (SRA) from the more than 60,000 available today to represent clinical isolates diversity and derived their CRISPR locus structure. The specific questions we tackled are: does MTC CRISPR locus contain additional spacers in addition to the 68 spacers ones described? What are the other patterns of diversity in CRISPR-Cas locus? What kind of underlying mechanisms of evolution can account for the observed diversity? Did the main lineages evolve similarly or are there CRISPR features specific of some lineages and/or sublineages? What is the most likely CRISPR sequence of tuberculosis most recent common ancestor (MRCA)?

\section{Results}

Exhaustive catalog of spacers in M. tuberculosis complex stricto sensu

We set up a method to identify not only variants of known spacers but also unknown spacers from $M$. tuberculosis CRISPR locus. Surprisingly, despite having explored more than 1000 sequencing data [35], we found no new spacers as compared to the 68 described previously for $M$. tuberculosis sensu stricto (excluding $M$. canettii or the new L0 and L8 lineages) [34]. The only new spacers that could be identified were found in $M$. canettii (data not shown). To identify whether this absence of new spacers could be due to a lack of sampling, we counted the cumulative number of spacers from the subset of isolates further described in this study upon 15 independent random samplings (Fig. 1). We found that the 68 known spacers were all sampled after having examined from 3 to 25 isolates. Our sampling was therefore one order of magnitude above the one that seems necessary to be exhaustive.

\section{Global structure of $M$. tuberculosis CRISPR}

We reconstructed the whole CRISPR loci for 198 clinical isolates representative of all $M$. tuberculosis diversity excluding $M$. canettii. CRISPR was almost always preceded by a complete set of cas genes, was followed by $R v 2813$, circumvented by one Direct Repeat sequence, DR0, at each of its border as can be seen for archetypal isolates 


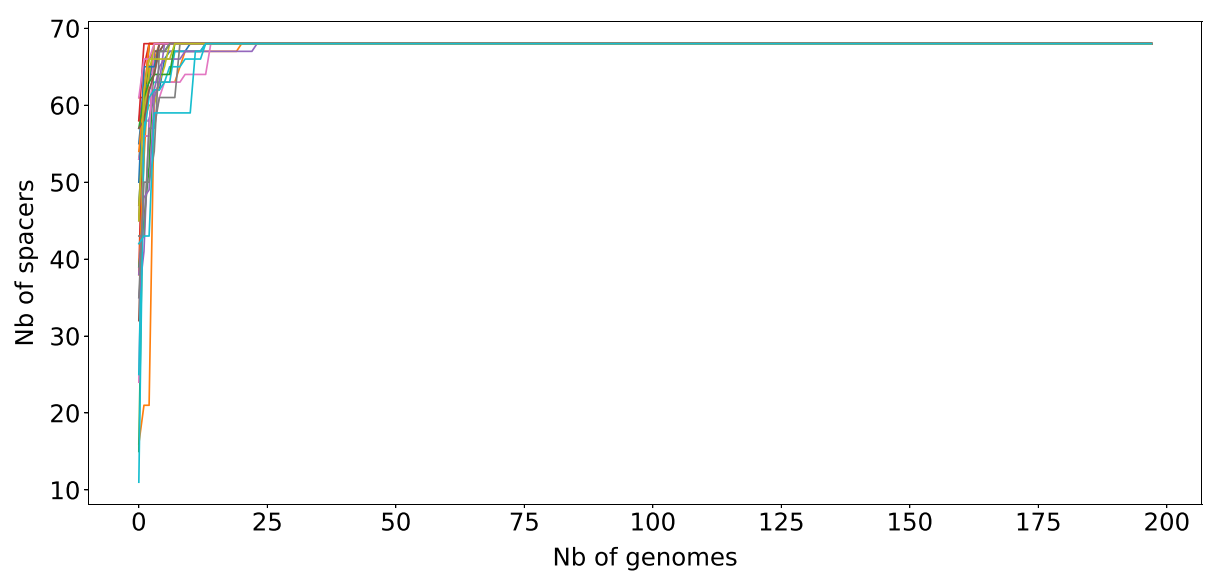

Fig. 1 Cumulative number of spacers along random sampling of our database. Footnote: * Our sampling shows that, with 200 SRR, we captured the entire diversity of spacers in Mycobacterium tuberculosis, with a maximum of 68 spacers

from each Lineage (Fig. 2). External DR0s are bordered by specific sequences, one of $48 \mathrm{bp}$ in length at the beginning of the locus, after Cas2, one of $148 \mathrm{bp}$ at the end of the locus, before Rv2813 (Supplementary file 1). These sequences are found in all isolates except in the case of large deletions (Supplementary file 2[IS6110 sheet]). Most of the time, the CRISPR-Cas locus includes one IS6110 copy as in the first isolate presented in Fig. 2 belonging to L1.1.1.6 (ERR751749), but it can go up to three copies or down to zero (Supplementary file 2[IS6110 sheet]). No other type of insertion sequence was ever discovered inside the region (data not shown).

The spacer sequences as well as those of the DR are always found in the same direction. Their order of succession is usually the expected one (the order of natural integers) although, as described below, various particular situations arise, for instance in case of duplications (Supplementary file 3). Duplications are identified not only by the order of

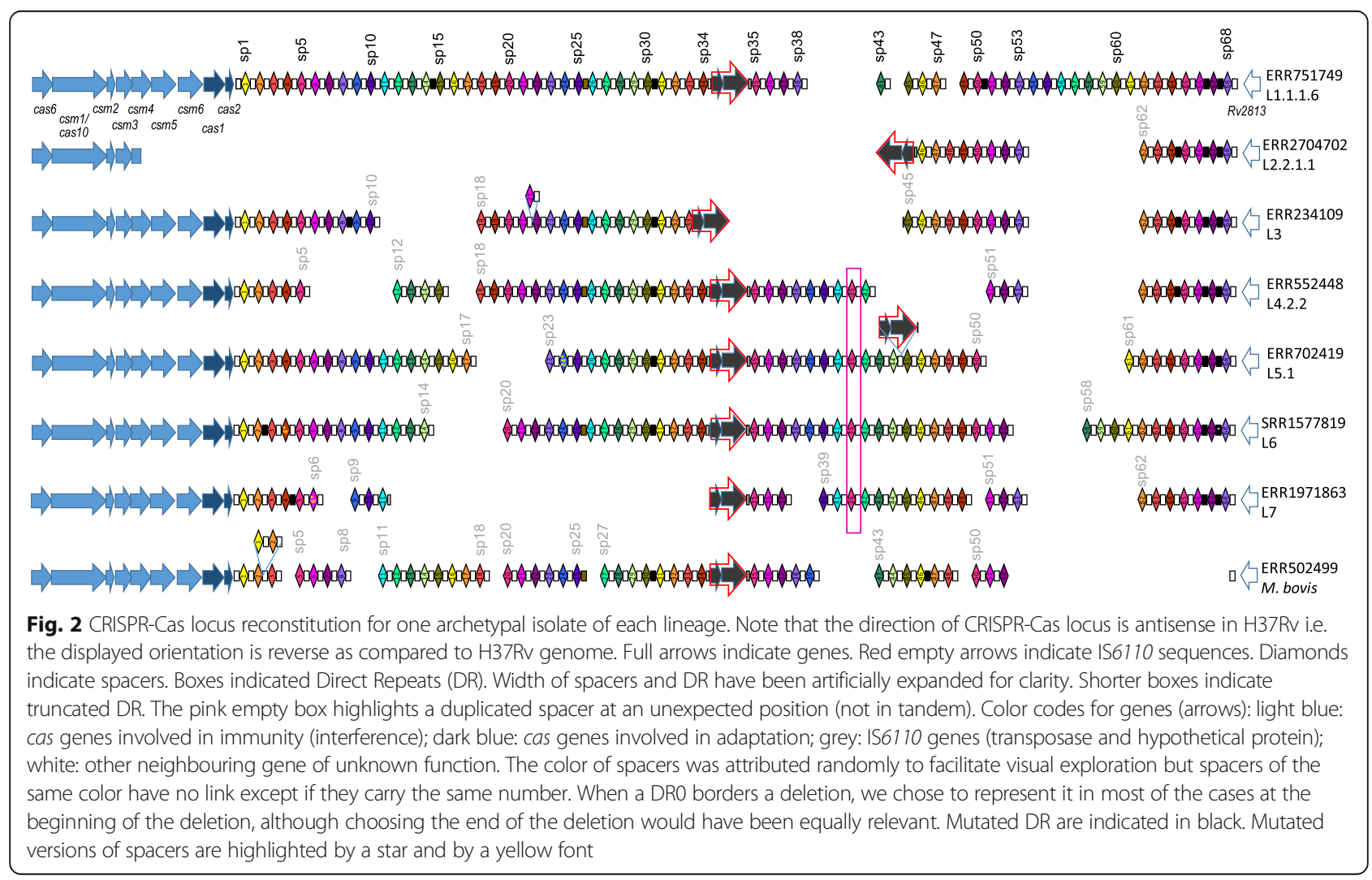


successive spacers, but also by the relatively higher reads quantity corresponding to the duplicated spacers. For instance, in an isolate belonging to L1.1.1.8 (ERR718201), while most spacers were found on an average of 27 reads, spacers 14 to 21 are found in 56 reads on average, which is approximately twice as much (Fig. 3). A notable exception in this isolate is spacer 16 that is found in only 31 reads. This in turn matches the fact that spacer 15 is half of the time followed by spacer 16 and the other half by spacer 17: in one of the two spacer 14-spacer 21 region, DVR16 has been deleted (Fig. 3).

Duplications occur in tandem most of the time. For instance, a second DVR21 is found after its normal copy in L3 isolates such as ERR234109, and an additional tandem DVR1-DVR2 is found downstream the standard pair in M. bovis ERR5022499 (Fig. 2). Other examples include DVR32 in ERR234197 (L1.1.3.1), DVR39 in ERR234248 (L2.1). This can be seen directly in the Illumina sequences, for instance for ERR234248, where many reads contain the end of 39 , followed by a DR0, followed by the beginning of another 39, which has no chance of happening, in such a repeated way, by chance due to random reading noise. A notable exception to the natural order of succession of spacer is the case of the spacer 35 , which can be found in the following two places: between 34 and 36 on the one hand, and after 41 on the other hand (Fig. 2, Supplementary file 4). Consequently, in most cases, although this is not the case of H37Rv and related isolates, there are two copies of 35 .

Another important and widely representative characteristic of MTC CRISPR locus is the presence of the IS6110 copy referenced in [29] and that shares the same orientation than the CRISPR, i.e. corresponding to a IS6110c (Fig. 2).

\section{Punctual variants in M. tuberculosis CRISPR}

Regarding intra-spacer diversity, we identified 20 spacers that harbored at least two variants, and concerned 48 (24\%) out of the 198 isolates explored (Supplementary file 2[spacer sheet]). These variants consisted mainly of SNP, although a deletion was found in spacer 24 in another dataset (genome ERR702419, lineage 5, data not shown). Interestingly, some of these variants are characteristic of specific lineages. For instance, a variant of spacer 38 is found in all isolates of lineage L1.1.1, one mutation is found in spacer 4 in all L6 isolates to which an additional one sometimes adds resulting in two possible variants. Two variants of spacer 6 characterize the endemic Abyssinian L7 isolates (Fig. 2, Supplementary file 2 and Supplementary file 5). The frequency of spacer variants in L2-L3-L4-L7 was relatively low (6 independent variants detected in 107 isolates, 5\%), as compared to L1 lineage (11 independent variants out of a selection of 55 isolates, $\sim 20 \%$ ) and lineage gathering animal isolates and L5 and L6 (7 independent variants for 34 isolates, 20\%).

Between two spacers, we have most of the time the DR0 sequence referenced in [34]. However, this rule is incomplete and not general. Punctual variants were identified. First of all, between spacers 30 and 31, there is always, whatever the lineage, a sequence that we coined DR2 and that has one punctual mutation as compared to DR0 (see sequence in Supplementary file 1). Similarly, there is always a DR4 variant repeat between spacers 66 and 67, and again a DR5 variant between spacers 67 and 68. This is true for all lineages, with the notable exception of a sublineage of L6, which has yet the DR10 variant (Fig. 2, Supplementary file 2[DR sheet]). Then, other types of variations were identified. For instance, between spacers 25 and 26, there are

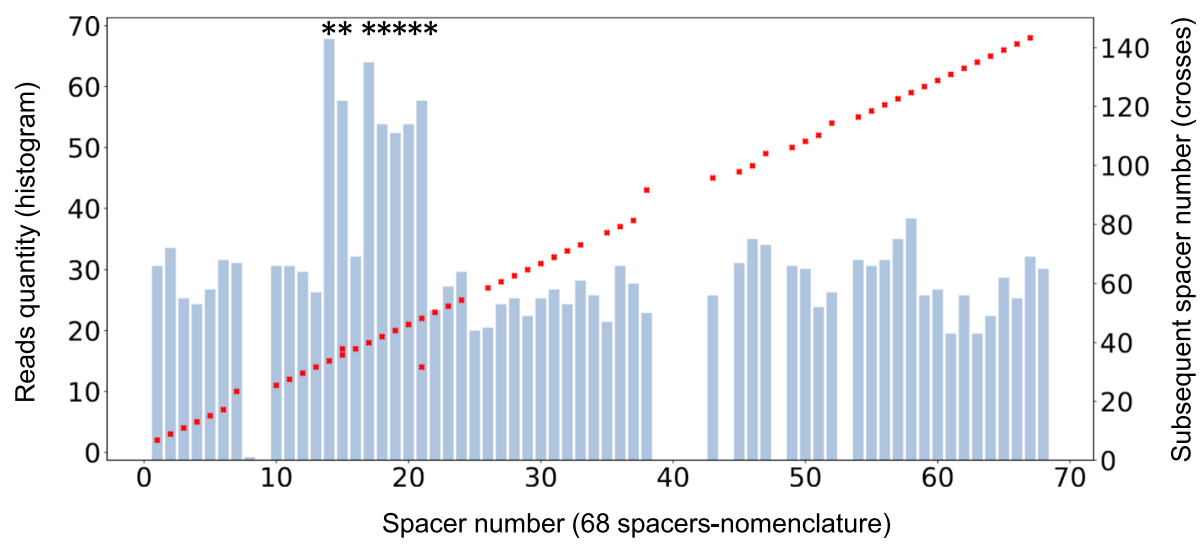

Fig. 3 Reads quantities and subsequent spacer number as convergent proofs of duplications, the case of ERR718201. Reads quantity are shown in blue. The number of the following spacer is shown in red crosses. *: spacers showing twice more reads than the average. Note that spacers 14,15,17,18,19,20,21 show twice more reads. Conversely spacer 21 is followed sometimes by spacer 22, sometimes by spacer 14 . This is compatible with a duplication of the region spacer $14-$ spacer21, with one repetition lacking spacer 16. Also note that the deletions are documented both by the absence of reads for the concerned spacers, and by a subsequent spacer (for instance spacer 9 deletion confirmed by the fact that spacer 8 is followed by spacer 10) 
always only the last 24 bp of DR0 (a sequence we name $\mathrm{DRb} 2$ ). Around the central IS6110c, between spacers 34 and 35 , the DR0 is split into two subsequences rDRa1 (upstream) and DRb1 (downstream). As expected due to IS6110 insertion characteristics, the concatenation of these two sequences is 3 bp larger than DR0 since 3 additional cytosines are present at each end of the insertion $[36,37]$. Yet, in a L5.1 isolate (ERR702419) where IS6110c inserted downstream spacer 44, IS6110c is preceded by the first $35 \mathrm{bp}$ of DR0 and followed by its 6 last bp, so that the duplicated target was this time $5 \mathrm{bp}$ in length (data not shown).

Some variants are shared over several but not all lineages or sublineages. For instance, DR6 is found between spacers 64 and 65, in all genomes of lineages L2 to L4 and only in those; DR10 is found between spacers 67 and 68 in L6. Similarly, the DR1 variant is found between 14 and 15 only in Sublineage L1.1.1, and never in Sublineage L1.1.2 or in any other lineage. These findings are consistent with $M$. tuberculosis phylogeny and allow to infer that the mutation in L1.1.1 occurred shortly after separation from the rest of the other L1 sublineages.

Other punctual variants affect a single isolate (Supplementary file 2[spacer and DR sheets] for isolates affected, Supplementary file $\mathbf{1}$ for their sequences). Each time, the size of the DR is respected (no indel, only the single nucleotide polymorphism) except for one case where a longer DR was found (data not shown). Altogether, these variants occurred all over the locus with no clear preferential subregion (Supplementary file 6).

\section{Large scale variations and IS6110 copies}

Large scale variations included on one hand deletions and on the other hand duplications. It should be noted that, at this stage, no inversion has been detected in MTC CRISPR.

Large-scale deletions were observed throughout the lineages, such as the one characterizing L2.2/Beijing sublineage that covers parts of $\operatorname{csm} 4$ to an IS6110 just before spacer 46 (\#36 in the old nomenclature). As in the case of this specific deletion, many deletions were flanked by an IS6110 insertion: the deletion between spacer 33 and spacer 45 in L3 isolates such as ERR234109, and the deletion between spacer 11 and spacer 35 in L7 isolates such as ERR1971863 (Fig. 2). To infer potential intermediates for these deletions, we searched for clinical isolates related to the one carrying deletions, and harbouring several IS6110 sequences. We found such evidence in Sublineage L4.1.2.1 (Haarlem sublineage). In this sublineage, a first set of isolates carry a 7 DVR- deletion adjacent to an IS6110 copy, namely between spacers 34 and the second copy of spacer 35 (for instance in ERR234259). A second set of clinical isolates (SRR5073877 and ERR552680) harbours two IS6110 copies, respectively the well-known one in the DR between spacers 34 and spacer 35 , and another one in the DR between spacer 41 and the second spacer 35 (Fig. 4). Interestingly, the borders of IS6110 insertion in ERR234259 corresponded well to the external borders of the two IS present in SRR5073877 and ERR552680. The left border consisted in the 17 first bp of DR0 ( 2 bp less only than the rDRa1 in the classical position), and the right border was the exact same 33-last nucleotides of DR0 than the one found at the right of the second insertion in SRR5073877 and ERR552680. The CRISPR version with the two copies shares many features with that carrying the deletion, suggesting that it could correspond to its ancestral stage of evolution (Fig. 4). The same observation in L4.1.2.1 was made independently in a study performed in Hanoi [38].

These large scale deletions involved cas flanking genes in 23/198 (12\%) of isolates, with two different borders in L2 isolates, two others in L4 and a third one in L3. In contrast, a single case was observed that affected $R v 2813$ (Supplementary file 2[IS6110 sheet]). We further explored this asymmetry using SITVIT2 2019 database ( $n=3852$ SITs): 290 SITs harbored a deletion of spacer \#1 (DVR2 in the new nomenclature) against 117 SITs with a deletion of spacer \#43 (DVR65 in the new nomenclature), $i$. e. three times more deletions on the cas genes side.

\section{Likely MRCA CRISPR of $M$. tuberculosis}

All variations we observed were concordant with the phylogeny of M. tuberculosis. We could thus infer the most likely structure of CRISPR locus of $M$. tuberculosis complex sensu stricto (without $M$. canettii), as well as its structure in all MRCA lineages. We found that global MRCA likely carried a full set of cas genes, a CRISPR with 69 spacers (the 68 spacers of different sequences + the repetition of spacer 35 ) interspersed mostly by DR0 except between spacers 25 and 26 (DRb2), spacers 30 and 31 (DR2), spacers 66 and 67 (DR4) and spacers 67 and 68 (DR5). An ancestral and central IS6110c was inferred to lie at the same place as the one occupied in H37Rv, i.e. between spacers 34 and 35 (Fig. 5). A deletion of DVR 54 to 61 characterized MRCA of lineages 2, 3,4 and 7 , which is not documented in the classical form of the spoligotype as these spacers are not belonging to its set of 43 spacers. Other deletions corresponded to the ones found in spoligotype- 43 format and used to define main sublineages. For instance, the deletion of spacers \#33-36 in the old nomenclature for L4/EuroAmerican lineage (previously referred to as $\mathrm{T}$ family) corresponds to the deletion of DVR43 to 50. Another example is the deletion of spacers \#29-32, presence of spacer \#33 and absence of spacer \#34 characteristic of Lineage 1 (previously referred to as EAI) [31] that corresponds to the deletion of DVR39 to 42, presence of 


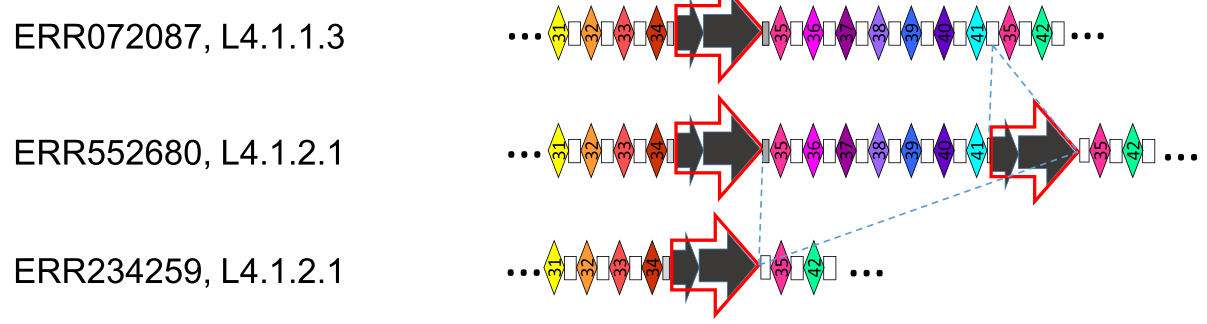

Fig. 4 IS-Driven CRISPR locus evolution mechanism suggested between L4.1.1.3 and L4.1.2.1. See Fig. 2 for legend. The three lines correspond to successive steps of evolution (starting from an ancestor with a structure identical to ERR072087, to the structure of ERR234259) according to a parsimonious reconstruction (see all individual CRISPR structures in Supplementary file 2)

DVR43 and absence of DVR44 (Fig. 5). Only L2 MRCA did not carry the well-known signature of Beijing isolates as L2 includes not only the Beijing L2.2 sublineage but also the L2.1 proto-Beijing sublineage [27]. Interestingly, this ancestor harbors an IS6110 insertion in one cas gene (namely csm6) but not at the border of the classical Beijing deletion. It also lacks DVR16 and DVR17.

\section{Discussion}

Thanks to our new Sequence Reads Archive-based genomic analysis pipeline, we explored the M. tuberculosis CRISPR sequences diversity in 198 clinical isolates representative of the MTC excluding $M$. canettii, which deserve new specific studies [39, 40]. These data show that $M$. tuberculosis CRISPR locus contains at most 69 spacers (68+one duplication), is not prone to inversions, evolves by duplication and deletions through recombination between DR, but also and primarily through insertion/deletions implicating IS6110, by homologous recombination, and independently of lineage. We detail below the support for these different kinds of mutations and inferences that can be drawn concerning the functionality of CRISPR-Cas locus.

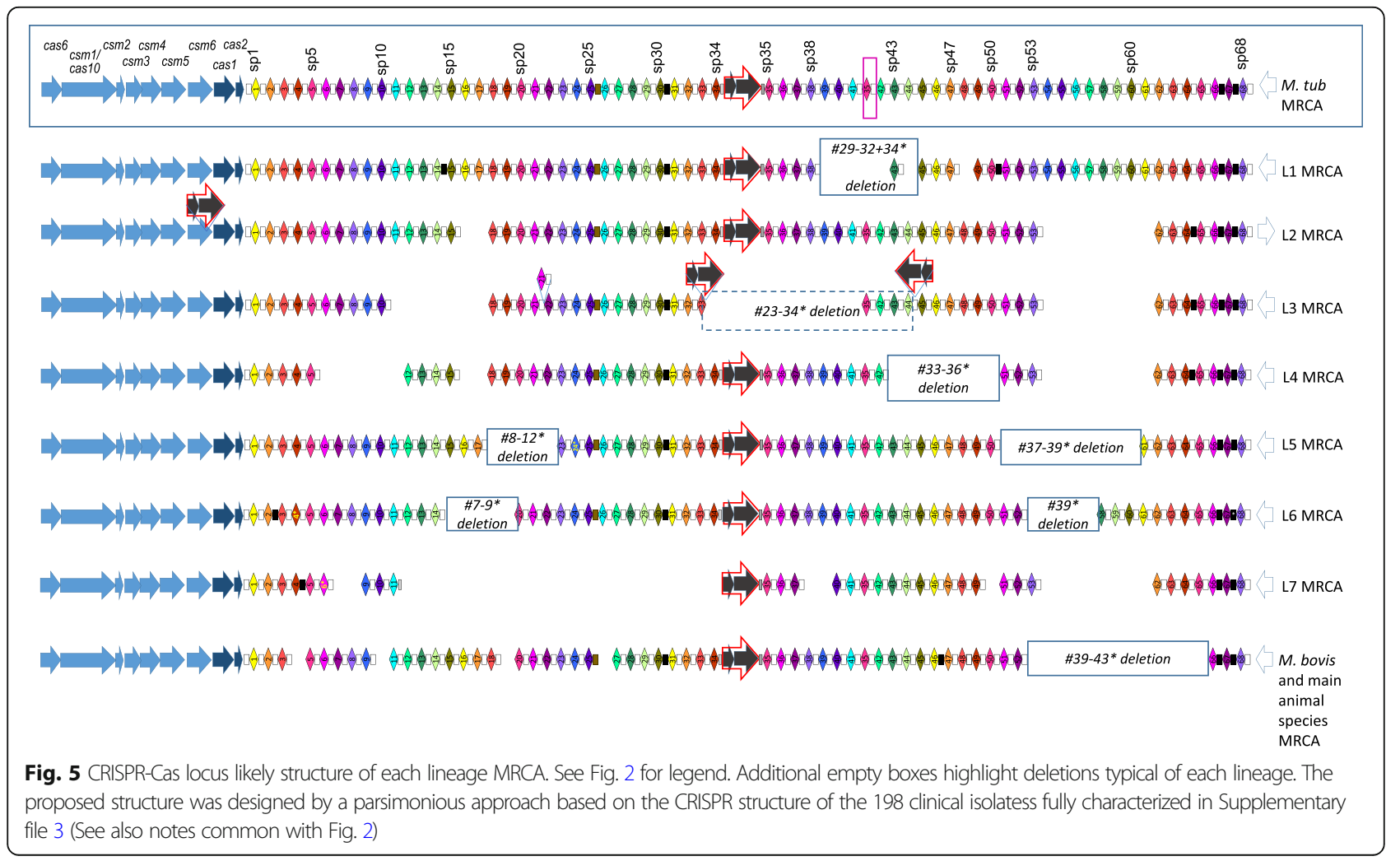


Evolutionary mechanisms of MTC CRISPR locus expansion Despite the absence of acquisition of new spacers, MTC CRISPR locus is of relative long size in many isolates (for instance, 4589 bp between Rv2813 and Rv2816c/ cas 2 in H37Rv). This relates to its ability to continue to expand using mechanisms other than classical CRISPR adaptation.

A first mechanism of MTC CRISPR size expansion, when considered as the distance between its two borders, is the integration of IS6110 insertion sequences (1355 bp). The most frequent insertion is found between spacers 34 and 35 as in H37Rv genome. Other IS6110 insertions were found along the whole MTC CRISPR locus, with up to two insertions in the CRISPR locus and three when considering the whole CRISPR-Cas locus. Other similar IS Sequences right next to or farther away, might be responsible for other homologous recombination mechanisms involving CRISPR.

The second CRISPR expansion mechanism identified in this overall review concerns duplications of DVR (DR + spacer). These duplications are of two main types. First of all, duplications can concern a single DVR and occur in tandem which was observed in 11 independent cases throughout our 198 samples. This type of tandem duplication concerns also several adjacent DVRs such as DVR1-2 in $M$. bovis or DVR14-15-16-17-18-19-20 in L1.1.1.7. Such multiple DVR duplications were observed 5 times in our sample, so that in total 16 independent events of tandem duplications were observed. The second type of duplications concerns DVR that are far away from their original position, a type we call "rearrangement duplications". This first concerns DVR35 located between DVR41 and DVR42 as already mentioned above and supposedly in MTC MRCA CRISPR. Other examples include a second copy of DVR3 found between DVR12 and 13 found in ERR036187 (L4.3.4.1), while in ERR234197 (L1.1.3.1), there is an additional copy of DVR38 between DVR55 and 56. In one instance, this concerned several adjacent DVRs: a second copy of DVR50-51-52-53 is found between DVR3 and 4 in ERR2245409 (L3.1.1). Altogether, this made a total of 4 independent rearrangement-duplications. The fact that rearrangement duplications are less common than standard duplications suggests that they occur less frequently and/or that they are less stable. If the stability of rearrangement duplications was low, there should be several cases of deletions between the two copies of DVR35 as they were likely already present in MTC MRCA. Yet, we observed no case where a deletion concerned solely the DVR between these two copies.

Overall, the proportion of genomes containing either several copies of IS6110 or a duplication of one of the forms listed above is important, showing that MTC CRIS $\mathrm{PR}$ is much more variable than what could be derived from a standard 43 spacers spoligotyping analysis. This is true not only for the in vitro but also for the in Silicobased acquisition of the spoligotype, as the blast procedure used in the current analytic tools (Spolpred, SpoTyping) only provides information on the presence or absence of a given spacer: there is nothing quantitative or locationrelated in these approaches [41, 42]. Hence, on one hand, the representation of the CRISPR locus through a simple barcode of presence/absence of individual spacers hides these quantitative and localization information, whereas on another hand, a more extensive description of the CRISPR locus including duplications, insertions, point mutations, provides useful information to classify and/or cluster clinical isolates. Such an information is advantageously correlated with the current SNPs based taxonomical system of MTC genomes and enhances our understanding of isolates evolution [20, 21, 26, 27, 43].

\section{Combined mechanism of CRISPR locus reduction: how does IS6110 contributes to the evolution of CRISPR locus in MTC?}

In addition to the undeniable expansion mechanisms mentioned above, CRISPR reduction mechanisms also coexist, which -to some extent- explain some of the spacer block deletions in MTC spoligotypes.

The first potential mechanism is the simple loss of spacer, for instance by recombination between two adjacent DRs. For instance, clinical isolate ERR1203071 of L4.8 lacks spacer 1. In place, it harbors a one nucleotide variant of the beginning sequence, a DR0 and spacer 2 . The principle of parsimony here tends to suggest that a recombination between the DR0 bordering spacer 1 led to this genotype. The same kind of recombination seems to occur on slightly higher number of DVR such as the DVR54-DVR61 deletion typical of L2-3-4-7. Recombination between normal, standard DRs would be favored compared to recombination between different DRs (one standard, one mutated).

We can now confidently argue that the second highly frequent mechanism that is at play for the largest suppressions of consecutive spacers, is an IS-linked three steps mechanism: (1) insertion or prior presence of a first copy of IS6110 (for instance that after spacer 34), (2) insertion of a second IS6110 copy at another location (e.g. in csm6 in the ancestor of L2, also seen in SRR1710060, see Supplementary file 2), and (3) recombination between the two IS6110 copies. This IS-mediated mechanism, that has been described in previous studies is a general mechanism, i.e. it happens independently of the lineage and is responsible of convergence in IS6110 copy numbers [44]. The final result is the change from $x$ to $x-1$ copies of IS6110, with the loss of all spacers between the two copies. This mechanism can be observed independently of lineages, for example, in lineage 4, in Haarlem (4.1.2.1): L4 ancestor 
has a single copy of IS between 34 and 35, then a second copy occurred in the ancestor of Haarlem L4.1.2 isolates as seen in ERR552680, between 41 and 35, and finally a deletion occurred leading to the loss of spacers 35 to 41 for some isolates such as ERR234259. It therefore seems reasonable to think that after the insertion after spacer 41, this copy of IS6110 has recombined with the one upstream of spacer 35 . This mechanism is also at work elsewhere in the Haarlem isolates between $\operatorname{csm} 5$ and spacer 34 and between $\operatorname{csm} 5$ and spacer 41 (Supplementary file 2).

IS6110 insertions can take place in spacers or in DR and it is not necessary for an IS to be in a DR to be able to recombine. For instance, in many L4.3 (LAM) clinical isolates where spacers 31 to 34 (\#21-\#24) are missing, the successive sequences of interest are: the beginning of spacer 31 (\#21), an IS6110c, DRb1 and spacer 35. The last three sequences of interest are found in the exact same order in undeleted isolates such as H37Rv. This suggests that an IS6110 copy was first inserted at the end of spacer 31, and that it later recombined with the one located between spacers 34 and 35 . This recombination did not modify the flanking sequences.

The orientation of the two IS6110 copies that recombined cannot always be derived due to the lack of the ancestral versions. Still in several cases, we could identify isolates related to the deleted ones, that carry the two IS6110 flanking the future deletion. This is true for the IS6110 insertions having led to the deletion described in Fig. 4. In that case, both insertions were in the reverse sense as compared to $\mathrm{H} 37 \mathrm{Rv}$ orientation and can be called IS6110c. In another case, the isolate with two IS6110 insertions is SRR5073887 (L4.4.1): it carries not only the standard IS6110c insertion between spacers 34 and 35 but also an IS6110 insertion in the sense direction at the 439th nucleotide of csm6. The deletion in ERR2653229 (also L4.4.1) flanked by the beginning of csm6 and DRb1 and spacer 35 with a sense IS6110 sequence in its middle (Supplementary file 2 [IS6110 sheet]) likely occurred through the recombination of these two IS although they lie in opposite orientations. This phenomenon was recently observed in several cases of IS6110 mediated deletions in L2 [45].

\section{Variants and problems in spoligotyping}

How does the CRISPR sequence diversity impact spoligotyping data? When performed in vitro, spoligotyping consists first in the amplification of the CRISPR locus using primers facing the outside of DR region, referred to as DRa and DRb, and second in the hybridization to probes attached at a specific position on a membrane or another support. CRISPR sequences variants may reduce the efficiency of the process, whether at the amplification or at the hybridization step. The presence of intermediate signals in spoligotyping or discrepant results between in silico and in vitro-based spoligotypes has been documented by several authors [46, 47]. We looked for intermediate signals corresponding to variants. In the case of L6 clinical isolates that carry a variant of spacer 4 (spacer 3 in spoligo-43 nomenclature), we found no evidence of such report in the literature and in our own data (data not shown). The same was true for spacer 38 (spacer 28 in spoligo- 43 nomenclature) found in L1.1.1 clinical isolates even if the mutation is relatively central in the probe (Supplementary file 5).

\section{Asymmetric variations affecting of MTC CRISPR-Cas locus} As described above, we identified punctual nucleotide mutations, duplications, IS insertions and deletions along CRISPR-Cas locus. CRISPR are oriented loci that acquire new spacers at the $5^{\prime}$ end relative to their transcription direction $[12,48]$. It may therefore be expected that variations do not affect symmetrically this locus. To explore and understand the consequences of this possibility, it is important to identify the orientation of the CRISPR locus in question. Using RNAseq data on H37Rv, Wei et al. showed that transcription occurs from spacer 1 towards spacer 68 [49]. We independently confirmed this observation by the exploration of independent RNAseq data from $[50,51]$ (Refregier et al. unpublished results). The orientation presented in this study is thus the functional one. According to classical CRISPR expansion mechanism, the introduction of new spacers occurs at the $5^{\prime}$ end of the locus, so that the most ancient DVR lies at its $3{ }^{\prime}$ end.

In contradiction with the remarkable feature that most ancient DR carry mutations in all isolates, no subregion exhibited a significantly higher punctual mutation rate (Supplementary file 6). The fact that the most ancient part of CRISPR locus does not carry a significantly higher number of punctual mutations as compared to parts that are more recent (spacer block deletions), may suggest that the time during which the locus expanded from spacer 68 to spacer 1 may be negligible as compared to the time between MTC MRCA and present, or that the CRISPR locus was transferred by lateral gene transfer in one single block from another environmental organism. Alternatively, the time of CRISPR locus expansion could have been quite long, however the pace of CRISPR locus SNPs mutations acquisition was very slow because of an extremely slow pace of MTC transmission. Demography and genetic drift could have been much more important for MTC evolution than selection in human populations [52]. Yet, the presence of mutations in several DR at the 3 ' end of the locus could also play a role in its stability.

In contrast, we detected an asymmetry concerning the loss of flanking sequences: it was apparently more 
frequent to have a loss of the beginning sequences of CRISPR, on the side of the cas genes (several independent isolates from L2 and from L4) than to have a loss of the ending sequences, i.e. on the side of $R v 2813$. All deletions implicating flanking sequences were bordered by an IS6110 sequence. Altogether, the asymmetry in deletion suggests either a more crucial role of the end of the CRISPR i.e. of gene $R v 2813$ and/or its neighbors, or asymmetric mechanisms favoring deletion on the cas gene side. This second possibility relates to IS6110 insertion frequency as IS are always involved in large deletions. Saying that IS6110 insertions are more likely on the cas gene side suggests either their lower impact on bacterial fitness, or a DNA superstructure that would favor IS insertions. Other IS exist in the genome that could also insert in a favorable region. Their presence in CRISPR region would be a sign that it is an integration hot spot. However, our script was designed to look only for insertion in cas gene that also lead to a deletion in the CRISPR in at least one of the explored sample.

IS other than IS6110 are unlikely to lead to any deletion. Even if our script may have overlooked non-IS6110 insertions, we did not encounter it in around 500 randomly sampled genomes. The question of cas gene locus being an integration hotspot of IS sequences needs other studies to be completely solved.

\section{Functionality of MTC CRISPR-Cas locus}

CRISPR-Cas loci are involved in two mechanisms: 1) adaptation by the integration of new spacers, usually taken from foreign DNA, at the $5^{\prime}$ end of CRISPR with the help of Cas1 and Cas2 proteins, and 2) immunity by the transcription of CRISPR locus, processing with the help of Cas6 protein in the case of type III-A CRISPRs, and degradation of DNA and/or RNA carrying protospacers, with the help of the crRNP (CRISPR RiboNucleoProtein complex), a complex involving the crRNA and other Cas proteins. By exploring the diversity of many genomes at the CRISPR locus, we are able to infer the effectivity of adaptation process. Regarding immunity, we can only state whether the necessary genes are present or not.

In the whole $M$. tuberculosis complex sensu stricto, we could find only the 68 spacers already present in the MRCA [34]. We found no evidence that a single clinical isolate has acquired a new spacer in the course of MTC evolution. This seems particularly surprising as most currently spreading isolates apart those from L2 still carry the full set of Cas genes including Cas1 and Cas 2 involved in CRISPR adaptation in other type III-A systems. This could be due to a mutation in $M$. tuberculosis ancestor that has abolished Cas1 and/or Cas2 functionality in the ancestor. Another reason could be that MTC, given its intracellular life-style, does simply not have the chance anymore to encounter foreign DNA such as phages or plasmids. These two phenomena could also be linked: a loss of functionality of Cas1 and Cas2 in the MRCA of all MTC could have fostered an adaptative change in lifestyle of the bacterium, i.e. from an environmental extracellular to a host-specialized intracellular life-style. Such an hypothesis could be supported by the evolution of the CRISPR locus of Vibrio cholerae, with observations that the recent pandemic strains have lost their ancestral CRIS PR locus [53] and (FX Weill, personal communication). Hence, the functionality of Cas1 and Cas2 of MTC remains to be explored.

Regarding immunity, this study only focused on the full presence or absence of cas genes without exploring in detail SNP variations. As stated previously, 23/198 (12\%) lacked at least part of the cas genes. Among these yet, all isolates still carried the cas6, cas10/csm1, csm2, and csm3 genes. This observation matches that made previously on CRISPR ${ }^{-}$clinical isolates [28]. Cas6 protein is involved in pre-crRNA processing. Cas10/Csm1 and $\mathrm{Csm} 3$ are the enzymes responsible for the catalytic activity of the crRNP $[54,55]$. Hence, regarding immunity, even if the spatial structure of the crRNP may be impaired by the absence of $\operatorname{csm} 4$ and/or $\operatorname{csm} 5$ in some isolates, it could remain possible that immunity occurs in all MTC isolates through the consecutive actions of Cas6 to process pre-crRNA and of Cas10/Csm1 and Csm3 to degrade DNA and/or RNA. The fact that none of the spacer is conserved in all isolates implies that, if immunity occurs, it does not always target the same DNA and/or RNA sequences.

\section{Global implication of CRISPR diversity for the understanding of MTC clinical isolates evolution}

In MTC, the CRISPR locus is a likely witness of a previous yet unknown evolutionary history of phage DNA invaders defense, whereas IS6110 is a specific MTC element that belongs to the IS3 family that, through transposition, also plays a permanent role in shaping MTC genomes [56]. The link between the two in evolutionary genomics remains poorly investigated until now. MTC genome actually contains a lot of other IS and transposases (88 genes retrieved in mycobrowser,https:// mycobrowser.epfl.ch/(https://mycobrowser.epfl.ch/) such as IS1081, IS1533, IS1547, IS1560), but IS6110 is the one with the largest number of copies in most isolates and especially in the reference isolate H37Rv [57]. IS1547 was previously shown to play a role in MTC evolution however it remains poorly investigated [58]. IS6110RFLP was the golden standard to define epidemiological clusters at the end of the nineties and stayed so during around 20 years, until it was replaced by MIRU-VNTR ${ }^{1}$ and more recently by Whole-Genome-Sequencing $[4,5$, 59-61] (for a recent review on evolution of TB molecular 
epidemiological methods, see also [1]). Previous results on IS6110 insertion sites have shown that independent IS6110 copy acquisition through transposition into hotspots was a common mechanism explaining convergence in IS6110 copy number in some of the MTBC sublineages $[44,62]$. A recent paper on the micro- and macroevolution of Lineage 2 of MTC in relation to IS6110 transposition also stresses the interest of such studies using WGS [45]. The role of the ipl (Insertion Preference Locus) was also stressed long time ago and showed consequences on the CRISPR locus [58,63,64], however no generalized observations on IS-CRISPR genomics dynamics had been done so far before this study.

\section{Conclusions}

Our study, by providing an in-depth reconstruction of the CRISPR locus of MTC in combination with IS6110 using short reads on around 200 genomes, improves our knowledge on the structure of the CRISPR locus and sheds new light on the general evolutionary mechanisms acting on MTC genomes through a first yet quantitatively limited analysis that combines CRISPR-IS combined evolutionary dynamics. By unveiling an unexpected genetic diversity of the CRISPR Locus on MTC, our study opens the way to new in-depth congruence analysis between SNP-based and repetitive sequence based MTC phylogenies. Such deeper knowledge on the natural history of tuberculosis will help us deciphering the most important key evolutionary events that shaped today's global and local MTC genomes population structure.

\section{Methods}

\section{Data collection}

One hundred ninety-eight $(n=198)$ Sequence Reads Archives obtained by paired-end sequencing with Illumina technology were selected from a local database of more than 3500 genome sequences based on their representativeness of M. tuberculosis lineages [35]. Namely, the following numbers of data were included for each lineage: 55 for Lineage 1, 20 for Lineage 2, 17 from Lineage 3, 60 from Lineage 4, 25 from Lineage 5, 7 from Lineage 6, 10 from Lineage 7, 1 from M. bovis, 1 from M. caprae, 1 from M. microti, 1 from M. pinnipedii. Data were downloaded as fasta files to decrease storage space as erroneous sequence will be ignored in the analytic steps.

\section{Identification and cataloging of CRISPR subsequences of interest}

We first included all known spacer sequences and the most common DR sequence, later referred to as DR0 [29].

We then looked for spacer variants by searching for patterns made up of the last 12 nucleotides of DR0 [29], followed by 10 to $70 \mathrm{bp}$, followed by the first $12 \mathrm{bp}$ of the DR0. The resulting subsequences were compared to the reference spacers to be declared either as a new spacer or a variant of a known spacer. We then used this enhanced catalogue of spacers to find DR variants, in the same way as above. The new DRs thus obtained were used for a second phase of discovery of spacers, as described above.

To the collection of different spacers and DR, we added the following subsequences of interest:

1) the beginning and end sequences of IS6110 and its reverse complement ( $40 \mathrm{bp}$ each time);

2) those corresponding to Rv2816c (Cas2 gene of the Cas locus) and $R v 2813 c$, reputed to border the CRISPR locus;

3) the sequences found between these bordering genes and first or last DR;

4) the beginning and the end of each Cas gene;

5) sequences in the neighbouring genes (Cas or others) when these sequences were found besides an IS6110 sequence during reconstruction -see below- (for more details; see [35].

An extended version of these sequences of interest is presented in Supplementary file 1.

The method is described and was fully validated using in silico simulated CRISPR in our methodological paper [35].

\section{Locus reconstruction}

An automated contig building method based on De Bruijn approach and referred to as CRISPRbuilder-TB (https:// github.com/cguyeux/CRISPRbuilder-TB) was set up to reconstruct large fragments of the CRISPR. CRISPR with IS6110 insertion could not directly be reconstructed as no read can overlap the full IS6110 sequence (1355 bp in length). Another reason for non-resolution of contigs is the existence of duplications: they lead to bifurcations in the de Bruijn graph. A specific search for duplications was included looking for patterns of the form $\mathrm{sp}$.l ${ }^{*}$ DRX*sp.(m), where $\mathrm{l} \geq \mathrm{m}$ (for more details see [35].

To facilitate the contigs concatenation, sequences were simplified by replacing each subsequence of interest by its name according to the catalogue described above. Final reconstruction taking into account IS6110 insertions was performed manually. In some samples, contig reconstruction was confirmed by retrieving the identity of the spacer downstream the last spacer of a duplication. When one side of the CRISPR could not be automatically recovered for instance due to an IS6110 insertion with a single end found in the catalog of CRIS PR locus sequences, a stepwise manual search for the neighbouring sequences was performed until recovery of the other IS6110 end. The $60 \mathrm{bp}$ sequence found nearby was labelled according to the gene it belongs to and its 
position, and it was added to the catalog of sequences of interest.

\section{Supplementary Information}

The online version contains supplementary material available at https://doi. org/10.1186/s12864-020-07178-6.

Additional file 1: Supplemental file 1. (doc) - Sequences of interest in CRISPR-Cas region of Mycobacterium tuberculosis complex.

Additional file 2: Supplemental file 2. (tab) - CRISPR reconstructions highlighting 1) global structure and position of IS6110 insertions ['IS6110' sheet]; 2) spacer variants ['spacer' sheet]; 3) DR variants ['DR' sheet]; 4) Duplicated DVR ['Duplic' sheet].

Additional file 3: Supplemental file 3. Exploration of read numbers for the reconstruction and identification of duplications, the case of ERR718197.

Additional file 4: Supplemental file 4. Confirmation of sp35 presence after spacer 41 in two Sequence runs from clinical isolatess belonging to $L 5$ and $L 2$ respectively

Additional file 5: Supplemental file 5. Spacer 4, spacer 6 and spacer 38 variants in parallel with 43-spacers spoligotyping probes

Additional file 6: Supplemental file 6. Cumulative punctual variant numbers 5DR variants + spacer variants) in groups of 5 successive DVR from DVR1-5 to the last three DVR (DVR66-68)

\section{Acknowledgements}

Laura Morel, Valentin Pohyer, Matthieu Petrou, three previous undergraduates students who contributed to the start of the MTC CRISPR genome projects in the team, are warmly acknowledged.

\section{Authors' contributions}

CG, GR, CS conceived the study. CG developed the pipeline, GC,GR,CS chose the genomes to be analyzed, GR and CG analyzed results helped by CS; GR, CS and CG wrote the manuscript, GR drew the Figures and built the Supplementary Tables; The author (s) read and approved the final manuscript.

\section{Funding}

This study was funded by CNRS (Centre National de la Recherche Scientifique) The University of Paris-Saclay and the University of Bourgogne Franche-Comte through recurrent research support to the research teams.

\section{Availability of data and materials}

All genomic data used were extracted from Public genome databases (NCBI or ENA archives). Computer Program specifically developed in this paper will be made freely available upon request to Christophe Guyeux (christophe. guyeux@univ-fcomte.fr).

Ethics approval and consent to participate

N.A. This study only uses publicly available data.

\section{Consent for publication}

All authors read and accepted the final submitted version.

\section{Competing interests}

The authors declare no competing interest

\section{Author details}

${ }^{1}$ Institute for Integrative Biology of the Cell (I2BC), CEA, CNRS, Univ. Paris-Sud, Université Paris-Saclay, cedex, 91198 Gif-sur-Yvette, France. ${ }^{2}$ FEMTO-ST Institute, UMR 6174 CNRS, DISC Computer Science Department, Univ. Bourgogne Franche-Comté (UBFC), 16 Route de Gray, 25000 Besançon, France.
Received: 13 December 2019 Accepted: 22 October 2020

Published online: 30 November 2020

\section{References}

1. Garcia De Viedma D, Perez-Lago L. The Evolution of Genotyping Strategies To Detect, Analyze, and Control Transmission of Tuberculosis. Microbiol Spectr. 2018;6(5):MTBP-0002-2016.

2. van Belkum A, et al. Short-sequence DNA repeats in prokaryotic genomes MMBR. 1998:62:275-93.

3. Jajou R, et al. Epidemiological links between tuberculosis cases identified twice as efficiently by whole genome sequencing than conventional molecular typing: a population-based study. PLoS One. 2018;13(4):e0195413.

4. Schurch $A C$, et al. High resolution typing by integration of genome sequencing data in a large tuberculosis cluster. J Clin Microbiol. 2010a;48(9):3403-6.

5. Schurch AC, et al. The tempo and mode of molecular evolution of Mycobacterium tuberculosis at patient-to-patient scale. Infect Genet Evol. 2010b;10(1):108-14.

6. The CryPTic Consortium, Allix-Beguec C, et al. Prediction of susceptibility to first-line tuberculosis drugs by DNA sequencing. N Engl J Med. 2018;379(15): 1403-15.

7. Mulholland CV, et al. Dispersal of Mycobacterium tuberculosis driven by historical european trade in the South Pacific. Front Microbiol. 2019. https:// doi.org/10.3389/fmicb.2019.02778.

8. Jansen $\mathrm{R}$, et al. Identification of genes that are associated with DNA repeats in prokaryotes. Mol Microbiol. 2002;43(6):1565-75.

9. Groenen PM, et al. Nature of DNA polymorphism in the direct repeat cluster of Mycobacterium tuberculosis; application for strain differentiation by a novel typing method. Mol Microbiol. 1993;10(5):1057-65.

10. Ishino $Y$, et al. Nucleotide sequence of the iap gene, responsible for alkaline phosphatase isozyme conversion in Escherichia coli, and identification of the gene product. J Bacteriol. 1987;169(12):5429-33.

11. Pourcel C, Salvignol G, Vergnaud G. CRISPR elements in Yersinia pestis acquire new repeats by preferential uptake of bacteriophage DNA, and provide additional tools for evolutionary studies. Microbiology. 2005;151(Pt 3):653-63

12. Barrangou $R$, et al. CRISPR provides acquired resistance against viruses in prokaryotes. Science. 2007:315(5819):1709-12

13. Couvin D, et al. CRISPRCasFinder, an update of CRISRFinder, includes a portable version, enhanced performance and integrates search for Cas proteins. Nucleic Acids Res. 2018a.

14. Couvin D, et al. Macro-geographical specificities of the prevailing tuberculosis epidemic as seen through SITVIT2, an updated version of the Mycobacterium tuberculosis genotyping database. Infect Genet Evol. 2018b.

15. Grissa I, et al. On-line resources for bacterial micro-evolution studies using MLVA or CRISPR typing. Biochimie. 2008;90(4):660-8.

16. Grissa I, Vergnaud G, Pourcel C. The CRISPRdb database and tools to display CRISPRs and to generate dictionaries of spacers and repeats. BMC Bioinformatics. 2007a;8:172

17. Grissa I, Vergnaud G, Pourcel C. CRISPRFinder: a web tool to identify clustered regularly interspaced short palindromic repeats. Nucleic Acids Res. 2007b;35(Web Server issue):W52-7.

18. Makarova KS, et al. An updated evolutionary classification of CRISPR-Cas systems. Nat Rev Microbiol. 2015;13(11):722-36.

19. Fabre $L$, et al. CRISPR typing and subtyping for improved laboratory surveillance of Salmonella infections. PLoS One. 2012;7(5):e36995.

20. Coll F, et al. A robust SNP barcode for typing Mycobacterium tuberculosis complex strains. Nat Commun. 2014a;5:4812

21. Coll F, et al. PolyTB: A genomic variation map for Mycobacterium tuberculosis. Tuberculosis (Edinb). 2014b;94(3):346-54.

22. Gagneux S. Host-pathogen coevolution in human tuberculosis. Philos Trans R Soc Lond Ser B Biol Sci. 2012;367(1590):850-9.

23. Hershberg $\mathrm{R}$, et al. High functional diversity in Mycobacterium tuberculosis driven by genetic drift and human demography. PLoS Biol. 2008;6(12):e311.

24. Blouin $Y$, et al. Significance of the identification in the horn of Africa of an exceptionally deep branching Mycobacterium tuberculosis clade. PLoS One. 2012;7(12):e52841

25. Ngabonziza, J.C.S., et al. An ancestral lineage of the Mycobacteriumtuberculosis complex discovered near the African Great Lakes, missing link between M. canettii and M. tuberculosis sensu stricto Nat Commun. 2020; https://doi.org/https://doi.org/10.1038/s41467-020-16626-6. 
26. Palittapongarnpim $P$, et al. Evidence for host-bacterial co-evolution via genome sequence analysis of 480 Thai Mycobacterium tuberculosis lineage 1 isolates. Sci Rep. 2018:8(1):11597.

27. Shitikov E, et al. Evolutionary pathway analysis and unified classification of east Asian lineage of Mycobacterium tuberculosis. Sci Rep. 2017;7(1):9227.

28. Freidlin PJ, et al. Structure and variation of CRISPR and CRISPR-flanking regions in deleted-direct repeat region Mycobacterium tuberculosis complex strains. BMC Genomics. 2017;18(1):168.

29. Kamerbeek J, et al. Simultaneous detection and strain differentiation of Mycobacterium tuberculosis for diagnosis and epidemiology. J Clin Microbiol. 1997;35(4):907-14.

30. Brudey $\mathrm{K}$, et al. Mycobacterium tuberculosis complex genetic diversity: mining the fourth international spoligotyping database (SpolDB4) for classification, population genetics, and epidemiology. BMC Microbiol. 2006; 6(6):23.

31. Filliol l, et al. Snapshot of moving and expanding clones of Mycobacterium tuberculosis and their global distribution assessed by spoligotyping in an international study. J Clin Microbiol. 2003;41(5):1963-70.

32. Comas I, et al. Genotyping of genetically monomorphic bacteria: DNA sequencing in Mycobacterium tuberculosis highlights the limitations of current methodologies. PLoS One. 2009:4(11):e7815.

33. Kato-Maeda M, et al. Strain classification of Mycobacterium tuberculosis: congruence between large sequence polymorphisms and spoligotypes. Int J Tuberc Lung Dis. 2011;15(1):131-3.

34. van Embden JDA, et al. Genetic variation and evolutionary origin of the direct repeat locus of Mycobacterium tuberculosis complex bacteria. J Bacteriol. 2000;182:2393-401. see also reviewed version: Guyeux et al, Plos Computational Biology 2020 (in press).

35. Guyeux, C., Sola, C. and Refrégier, G. Exhaustive reconstruction of the CRISPR locus in M. tuberculosis and corresponding reviewed version in Plos Computational Biology (2020, in press) complex using short reads BioRxiv. 2019. https://doi.org/10.1101/844746.

36. Gonzalo-Asensio J, et al. New insights into the transposition mechanisms of IS6110 and its dynamic distribution between Mycobacterium tuberculosis complex lineages. PLoS Genet. 2018;14(4):e1007282.

37. Thierry D, et al. IS6110, an IS-like element of Mycobacterium tuberculosis complex. Nucleic Acids Res. 1990;18:188.

38. Maeda S, et al. Genotyping of Mycobacterium tuberculosis spreading in Hanoi, Vietnam using conventional and whole genome sequencing methods. Infect Genet Evol. 2020;78:104107.

39. Supply P, et al. Genomic analysis of smooth tubercle bacilli provides insights into ancestry and pathoadaptation of Mycobacterium tuberculosis. Nat Genet. 2013;45(2):172-9.

40. van Soolingen $D$, et al. A novel pathogenic taxon of the Mycobacterium tuberculosis complex, Canetti: characterization of an exceptional isolate from Africa. Int J Syst Bacteriol. 1997:47(4):1236-45.

41. Coll F, et al. SpolPred: rapid and accurate prediction of Mycobacterium tuberculosis spoligotypes from short genomic sequences. Bioinformatics. 2012;28(22):2991-3.

42. Xia E, Teo YY, Ong RT. SpoTyping: fast and accurate in silico Mycobacterium spoligotyping from sequence reads. Genome Med. 2016;8(1):19.

43. Stucki D, et al. Mycobacterium tuberculosis lineage 4 comprises globally distributed and geographically restricted sublineages. Nat Genet. 2016; 48(12):1535-43.

44. Roychowdhury T, Mandal S, Bhattacharya A. Analysis of IS6110 insertion sites provide a glimpse into genome evolution of Mycobacterium tuberculosis. Sci Rep. 2015;5:12567.

45. Shitikov E, et al. The role of IS6110 in micro- and macroevolution of Mycobacterium tuberculosis lineage 2. Mol Phylogenet Evol. 2019;139: 106559.

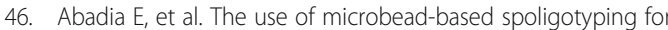
Mycobacterium tuberculosis complex to evaluate the quality of the conventional method: providing guidelines for quality assurance when working on membranes. BMC Infect Dis. 2011;11:110.

47. Meehan CJ, et al. The relationship between transmission time and clustering methods in Mycobacterium tuberculosis epidemiology. EBioMedicine. 2018;37:410-6.

48. Makarova KS, Wolf YI, Koonin EV. Classification and nomenclature of CRISPRCas systems: where from Here? CRISPR J. 2018;1(5):325-36.
49. Wei J, et al. The Mycobacterium tuberculosis CRISPR-associated Cas1 involves persistence and tolerance to anti-tubercular drugs. Biomed Res Int. 2019;2019:7861695.

50. Ignatov DV, et al. Dormant non-culturable Mycobacterium tuberculosis retains stable low-abundant mRNA. BMC Genomics. 2015;16:954.

51. Rodriguez JG, et al. Global adaptation to a lipid environment triggers the dormancy-related phenotype of Mycobacterium tuberculosis. MBio. 2014; 5(3):e01125-14.

52. Pepperell $\mathrm{C}$, et al. Bacterial genetic signatures of human social phenomena among M. tuberculosis from an Aboriginal Canadian population. Mol Biol Evol. 2010;27(2):427-40.

53. Weill FX, et al. Genomic history of the seventh pandemic of cholera in Africa. Science. 2017;358(6364):785-9.

54. Kazlauskiene $\mathrm{M}$, et al. A cyclic oligonucleotide signaling pathway in type III CRISPR-Cas systems. Science. 2017:357(6351):605-9.

55. Samai $P$, et al. Co-transcriptional DNA and RNA cleavage during type III CRIS PR-Cas immunity. Cell. 2015:161(5):1164-74.

56. Thabet S, Souissi N. Transposition mechanism, molecular characterization and evolution of IS6110, the specific evolutionary marker of Mycobacterium tuberculosis complex. Mol Biol Rep. 2017:44(1):25-34.

57. Cole ST, et al. Deciphering the biology of Mycobacterium tuberculosis from the complete genome sequence. Nature. 1998;393(6685):537-44.

58. Fang Z, et al. Characterization of IS1547, a new member of the IS900 family in the Mycobacterium tuberculosis complex, and its association with IS6110. J Bacteriol. 1999a;181(3):1021-4.

59. Supply $P$, et al. Proposal for standardization of optimized mycobacterial interspersed repetitive unit-variable-number tandem repeat typing of Mycobacterium tuberculosis. J Clin Microbiol. 2006:44(12):4498-510.

60. van Embden JD, et al. Strain identification of Mycobacterium tuberculosis by DNA fingerprinting: recommendations for a standardized methodology. J Clin Microbiol. 1993:31(2):406-9.

61. van Soolingen, D., Kremer, K. and Hermans PW. Molecular Epidemiology: Breakthrough Achievements and Future Prospects. In: Amadeo, editor, Tuberculosis 2007: from basic sicence to patient care Amadeo; 2007. p. Chapter 9.

62. Dale JW, et al. Evolutionary relationships amongst isolates of Mycobacterium tuberculosis with few copies of IS6110. J Bacteriol. 2003:185(8):2555-62.

63. Fang Z, et al. IS6110-mediated deletions of wild-type chromosomes of Mycobacterium tuberculosis. J Bacteriol. 1999b;181(3):1014-20.

64. Fang Z, Forbes KJ. A Mycobacterium tuberculosis IS6110 preferential locus (ipl) for insertion into the genome. J Clin Microbiol. 1997;35(2):479-81.

\section{Publisher's Note}

Springer Nature remains neutral with regard to jurisdictional claims in published maps and institutional affiliations.

Ready to submit your research? Choose BMC and benefit from:

- fast, convenient online submission

- thorough peer review by experienced researchers in your field

- rapid publication on acceptance

- support for research data, including large and complex data types

- gold Open Access which fosters wider collaboration and increased citations

- maximum visibility for your research: over $100 \mathrm{M}$ website views per year

At $\mathrm{BMC}$, research is always in progress.

Learn more biomedcentral.com/submissions 